\title{
Jet-Evolution in the Quark-Gluon Plasma from RHIC to the LHC
}

\author{
S. Domdey ${ }^{a}$, B.Z. Kopeliovich ${ }^{a, b}$, H.J. Pirner ${ }^{a}$ \\ a) Institute for Theoretical Physics, University of Heidelberg, Germany \\ b) Departamento de Física, Universidad Técnica Federico Santa María; \\ Instituto de Estudios Avanzados en Ciencias e Ingeniería; \\ Centro Científico-Tecnológico de Valparaíso, Casilla 110-V, Valparaíso, Chile \\ The observed suppression of high- $p_{\perp}$ hadrons allows different explana- \\ tions. We discuss two possible scenarios: In scenario 1, parton energy \\ loss from scattering in the hot medium is complemented by final state in- \\ teractions in the resonance matter. Scenario 2 has an enhanced transport \\ parameter $\hat{q}$ which is fitted to RHIC data. For LHC, the two scenarios \\ lead to very different predictions for the nuclear modification factor of \\ hadrons. In addition, jet reconstruction allows more specific tests of the \\ mechanisms responsible for jet quenching. We calculate the distribution \\ of partons inside a jet and find different results for the two scenarios.
}

\section{Introduction}

Investigations of the hot medium produced in heavy-ion collisions largely suffer from both its short life time and the small production rate of hard probes. Proceeding from experiments at RHIC to LHC, these features will be significantly improved. In particular, the increase in energy at LHC will lead to a higher temperature compared to RHIC which corresponds to a longer life time. Secondly, full jet reconstruction provides more differential information on the mechanisms of final state interactions compared to spectra of leading hadrons.

The large suppression of leading hadrons as observed by PHENIX and STAR coll. [1] (see also [2]) has triggered large interest in final state interactions in the hot medium produced in heavy-ion collisions. Theoretical models have focussed mostly on the energy loss of fast partons during their propagation through the dense medium. Many discussions 
of the range of mechanisms at work at RHIC and possibly at LHC can be found in the literature (see for example [3]).

In the picture of partonic energy loss, hadronization takes place outside of the hot zone and is not affected by the medium. Special features may develop due to the nonabelian nature of QCD. Coherent radiative energy loss may be important due to the radiated gluon interacting with the medium. This explanation corresponds to a less pronounced suppression of heavy charmed quark/hadrons in contrast to experimental results [4].

Gluon radiation undoubtedly plays an important role for parton evolution at RHIC and the LHC. Typical virtualities of partons are around $Q=20 \mathrm{GeV}$ and $Q=100$ $\mathrm{GeV}$, respectively. The resulting parton showers extend over several fm and evolve in the medium. Therefore, a separation of this shower from the propagation of partons in the medium seems to be unrealistic. From our perspective, however, there are no strong indications for coherence yet, because of the finite size of the medium which blurs the characteristic dependence of the energy loss on the square of the path length [5, 6, 7, 8, 9].

In our view, it is pragmatic to explore parton evolution interleaving radiation and interaction with the medium incoherently. Once the parton dynamics has been settled, measurements of the high- $p_{\perp}$ hadrons and/or jets can be used as a tool to investigate the properties of the quark gluon plasma. The further hypothesis of a strongly interacting quark gluon plasma (sQGP) [10] can be tested. This idea underlies models with large energy loss parameters $\hat{q}$, but has not received so much backing from lattice simulations where the quark gluon coupling constant was monitored in the region $0.5<\alpha_{s}<1$ at most [11.

A typical shower at RHIC evolves from a high virtuality around $Q=20 \mathrm{GeV}$ to a low perturbative scale $Q_{0}=1.5 \mathrm{GeV}$ and lasts about $\tau_{\text {evo }}=2 \mathrm{fm}$. In comparison, for longitudinal expansion with an initial temperature of $T_{0}=300 \mathrm{MeV}$ at $\tau_{0}=0.5 \mathrm{fm}$, one may estimate a plasma lifetime of $\tau_{c}=3.3 \mathrm{fm}$ [For details on these estimates, see Eq. (18) and Eq. (19).]. From these two time estimates, we find

$$
\tau_{\mathrm{evo}} \approx \tau_{c} \text {. }
$$

The nonperturbative part of hadronization involves the decay of the prehadrons or resonances at the preconfinement scale $Q_{0}$ where e.g. the vector meson resonances decay into $4-5$ pions. Therefore, it is very probable at RHIC that at the end of the evolution resonances interact with the remainder of the quark-gluon plasma, i.e. with hadronic resonance matter [26]. For $T \leq T_{c}$, the equation of state of hadronic matter can well be described by a hadron resonance gas with an initial density $n_{\text {res }} \approx T_{c}^{3}[12$. For our purposes, we neglect the slow cool-down of resonance matter below $T_{c}$. Such an interaction may be 
described by a hadronic theory with cross sections slightly larger than hadronic cross sections in vacuum. In general, hadronic resonances have radii which are larger than hadronic ground states. Therefore, one expects that $q \bar{q}$-resonances have larger cross section than the $q \bar{q}$-ground states. Because of these large cross sections, newly formed prehadrons are absorbed. We think that final state effects of these resonances with the hot resonance gas play a decisive role in the observed suppression of hadrons in RHIC experiments.

We therefore would like to advocate a comparison of two scenarios.

- Scenario 1 uses an estimate of $\hat{q}=0.5 \mathrm{GeV}^{2} / \mathrm{fm}$ for the jet transport parameter at RHIC. This scenario underestimates the suppression when only parton energy loss is taken into account. Additionally, we consider the absorption of the prehadrons in the resonance gas, leading to a similar suppression as observed at RHIC.

- Scenario 2 considers large parton energy loss solely and tunes up this value to $\hat{q}=4$ $\mathrm{GeV}^{2} / \mathrm{fm}$ in order to accomodate RHIC data.

Phenomenologically, we propose to test both scenarios on LHC data. For this purpose, we extrapolate both scenarios to $\mathrm{Pb}+\mathrm{Pb}$ collisions at $\mathrm{LHC}$ with $\sqrt{s}=5.5 \mathrm{TeV}$, a virtuality of $Q=100 \mathrm{GeV}$ and an initial temperature of $T_{0}=500 \mathrm{MeV}$. In the LHC case, the average time for the evolution from the initial high virtuality to a hadronic scale $Q_{0}$ is much longer than the plasma life time and the size of the hot interaction zone,

$$
\tau_{\text {evo }}>\tau_{c}
$$

Therefore we expect that resonances do not play an important role there.

Another important feature of LHC experiments is the possibility of full jet reconstruction up to very small momenta. Therefore we will also make predictions for jet compositions down to very small momentum fractions which govern the jet multiplicity. In both scenarios, the respective temperatures and virtualities control the parametric dependence of the input data to the modified DGLAP equations we use. The main uncertainty concerns the question whether final state absorption is important. Should we describe RHIC physics in the pure energy loss scenario 2 or in the mixed scenario 1, where nonperturbative features of resonance interactions occur together with perturbative parton interactions during hadronization? We claim that LHC will tell which scenario is preferred with its forthcoming experimental results at the end of this year. 


\section{Formalism and Modified Evolution Equations}

Hard processes in a heavy-ion collision lead to the production of partons with high virtuality and high energy. These partons will successively radiate gluons to reduce their virtuality and become on mass-shell. This leads to a parton shower and scaling violations in the vacuum jet fragmentation functions $D_{i}^{v}\left(x, Q^{2}\right)$ described by the DGLAP equations [13]

$$
\frac{\partial}{\partial \ln Q^{2}} D_{i}^{v}\left(x, Q^{2}\right)=\frac{\alpha_{s}\left(Q^{2}\right)}{2 \pi} \int_{x}^{1} \frac{\mathrm{d} z}{z} P_{j i}(z) D_{j}^{v}\left(\frac{x}{z}, Q^{2}\right) .
$$

In Ref. [35], some of the authors have developed a model for the parton cascade that includes scattering off the partons in the QGP. In this model, we modified the DGLAP equations by an additional scattering term.

In this section, we repeat the formalism for our further considerations. We consider both quarks and gluons as hadronizing partons. Indices on the fragmentation functions and the splitting functions indicate the respective parton species.

The formation of a parton shower does not happen instantaneously, but requires a certain time. The relevant time is the time a virtual state needs to evolve in virtuality from $Q^{2}$ to $Q^{2}+\mathrm{d} Q^{2}$,

$$
\mathrm{d} \tau=\frac{E}{Q^{2}} \frac{\mathrm{d} Q^{2}}{Q^{2}}
$$

where $E$ is the energy of the parton and $Q$ its virtual mass. The time a parton needs to reduce its virtuality from the starting scale $Q_{i} \simeq E$ to the hadronization scale $Q_{0} \simeq 1 \mathrm{GeV}$

can then be estimated as $\tau_{\text {evo }}=\int \mathrm{d} \tau \approx \frac{E}{Q_{0}^{2}}-\frac{1}{E}$. Thus, even though a high energy parton with large virtuality will reduce its virtuality rapidly, the overall lifetime is considerable and of the order of several fermi.

Furthermore, the plasma life time $\tau_{c} \simeq \tau_{0}\left(T_{0} / T_{c}\right)^{3}$ (corresponding to the cool-down from the initial temperature to the critical temperature) may be long and of similar magnitude. Consequently, the parton shower overlaps in time with the plasma phase. Therefore, splitting and scattering processes have to be treated in a common framework. During the time a fast parton reduces its virtuality by building up the parton shower, it experiences scatterings with gluons of thermal mass $m_{s}$ in the plasma. Therefore, we include a scattering term $S\left(x, Q^{2}\right)$ into the DGLAP evolution for the fragmentation function $D^{m}\left(x, Q^{2}\right)$ in the medium. The resulting evolution equation combines radiation and scattering:

$$
\frac{\partial D^{m}\left(x, Q^{2}\right)}{\partial \ln Q^{2}}=\frac{\alpha_{s}\left(Q^{2}\right)}{2 \pi} \int_{x}^{1} \frac{\mathrm{d} z}{z} P(z) D^{m}\left(\frac{x}{z}, Q^{2}\right)+S\left(x, Q^{2}\right) .
$$

For the construction of the scattering term we estimate the relative importance of 
scatterings with the help of the scattering mean free path $\lambda=(n \sigma)^{-1}$ by

$$
\frac{\mathrm{d} \tau}{\mathrm{d} \lambda}=\frac{E_{\text {in }}}{Q^{2}} n \frac{\mathrm{d} \sigma}{\mathrm{d} q_{\perp}^{2}} \mathrm{~d} q_{\perp}^{2} \frac{\mathrm{d} Q^{2}}{Q^{2}} .
$$

Two contributions to scattering have to be taken into account: A gain term for scattering from a higher energy fraction to the given energy fraction $x$ and a loss term for scattering from $x$ to lower energy fractions. Consequently, the energy $E_{\text {in }}$ of the incoming parton (before the scattering takes place) is different in the gain and the loss term (c.f. Eq. (7)).

Scattering makes the fast parton lose energy, which is absorbed as recoil energy $q_{\perp}^{2} /\left(2 m_{s}\right)$ by the plasma parton. In our treatment, we allow only soft scattering in the $t$-channel, i.e. small relative transverse momentum $q_{\perp}^{2} \sim m_{D}^{2}$, such that the change of longitudinal momentum fraction $\Delta x=y-x=q_{\perp}^{2} /\left(2 m_{s} E\right)$ is small. The Debye mass $m_{D}$ of the gluon and the thermal mass $m_{s}$ are related as $2 m_{s}^{2}=m_{D}^{2}$ [16] and $m_{D} \approx 3 T$ [34]. In contrast to splitting processes, which lead to a multiplicative change of the energy fraction of the parton, we model soft scattering processes by shifts to smaller energy fractions.

Scattering is included in the evolution equation in a similar way as radiation. The "scattering probability" Eq. (6) is folded with the fragmentation functions and gain and loss term are subtracted from each other. The additional scattering term in the evolution equation has the following form

$$
\begin{gathered}
S\left(x, Q^{2}\right)=\frac{E}{Q^{2}} \bar{n} \int_{x}^{1} \mathrm{~d} w \\
\times \delta\left(w-x-\frac{q_{\perp}^{2}}{2 m_{s} E}\right) .
\end{gathered}
$$

We then expand in powers of $q_{\perp}^{2} /\left(2 m_{s} E\right)$ and drop terms of second order and higher:

$$
\begin{aligned}
S\left(x, Q^{2}\right) & =\frac{E}{Q^{2}} \bar{n} \int \mathrm{d} q_{\perp}^{2} \frac{\mathrm{d} \bar{\sigma}}{\mathrm{d} q_{\perp}^{2}}\left[\left(x+\frac{q_{\perp}^{2}}{2 m_{s} E}\right) D\left(x+\frac{q_{\perp}^{2}}{2 m_{s} E}, Q^{2}\right)-x D\left(x, Q^{2}\right)\right] \\
& \simeq \frac{\bar{n}}{2 m_{s} Q^{2}} \int \mathrm{d} q_{\perp}^{2} \frac{\mathrm{d} \bar{\sigma}}{\mathrm{d} q_{\perp}^{2}} q_{\perp}^{2}\left(D\left(x, Q^{2}\right)+x \frac{\partial D}{\partial x}\left(x, Q^{2}\right)\right) \\
& \simeq \frac{\bar{n} \bar{\sigma}\left\langle q_{\perp}^{2}\right\rangle}{2 m_{s} Q^{2}}\left(D\left(x, Q^{2}\right)+x \frac{\partial D}{\partial x}\left(x, Q^{2}\right)\right)
\end{aligned}
$$

Such an expansion is meaningful for small momentum transfer, $q_{\perp}^{2} /\left(2 m_{s} E\right) \ll 1$ and $x+$ $q_{\perp}^{2} /\left(2 m_{s} E\right)<1$ as we expect them for small momentum transfers $q_{\perp}^{2} \sim m_{D}^{2}$ and large jet energies. Remarkably, the scattering term does not depend on the jet energy $E$ in this approximation. 
In the expression above, $\bar{n} \frac{\mathrm{d} \bar{\sigma}}{\mathrm{d} q_{\perp}^{2}}$ denotes the weighted sum of possible scattering channels. Depending on the incident parton, these can be quark-quark, quark-gluon and gluon-gluon scatterings:

$$
\bar{n} \frac{\mathrm{d} \bar{\sigma}}{\mathrm{d} q_{\perp}^{2}}=\frac{16}{\pi^{2}} \zeta(3) T^{3} \frac{2 \pi \alpha_{s}\left(Q^{2}\right)^{2}}{\left(q_{\perp}^{2}+m_{D}^{2}\right)^{2}} \times\left\{\begin{array}{cc}
\left(1+\frac{7}{8}\right)=\frac{15}{8} & \text { incident quark } \\
\left(\frac{9}{4}+\frac{63}{32}\right)=\frac{135}{32} & \text { incident gluon }
\end{array} .\right.
$$

Interestingly, the quantity appearing in the numerator in Eq. (8) is the jet transport parameter [5]

$$
\hat{q} \simeq \bar{n} \bar{\sigma}\left\langle q_{\perp}^{2}\right\rangle
$$

which describes the mean acquired transverse momentum per unit length (For a recent discussion of $\hat{q}$ see [18]).

For an estimate of the numerical value of $\hat{q}$ associated with this expression, we use

$$
\left\langle q_{\perp}^{2}\right\rangle \simeq m_{D}^{2}, \quad \hat{q} \simeq \frac{16}{\pi^{2}} \zeta(3) T^{3} \times 2 \pi \alpha_{s}^{2}\left(Q_{0}^{2}\right) \times\left\{\begin{array}{cl}
\left(1+\frac{7}{8}\right)=\frac{15}{8} & \text { incident quark } \\
\left(\frac{9}{4}+\frac{63}{32}\right)=\frac{135}{32} & \text { incident gluon }
\end{array}\right.
$$

The scattering term is most relevant at small virtualities $Q \simeq Q_{0}$ and consequently we use $\alpha_{s}\left(Q_{0}\right)$ to arrive at an upper boundary for $\hat{q}$. Explicitly, Eq. (11) gives $\hat{q} \simeq 0.5 \mathrm{GeV}^{2} / \mathrm{fm}$ for a temperature $T=0.3 \mathrm{GeV}$ for $\mathrm{RHIC}$ and $\hat{q}=5.2 \mathrm{GeV}^{2} /$ fm for $T=0.5 \mathrm{GeV}$ corresponding to LHC. To fit the experimental data with parton energy loss exclusively, we introduce an enhancement factor $K$ in the scattering term. Then the scattering term reads

$$
S\left(x, Q^{2}\right)=K \frac{\bar{n} \bar{\sigma}\left\langle q_{\perp}^{2}\right\rangle}{2 m_{s} Q^{2}}\left(D^{m}\left(x, Q^{2}\right)+x \frac{\partial D^{m}}{\partial x}\left(x, Q^{2}\right)\right) .
$$

The value of $\hat{q}=0.50 \mathrm{GeV}^{2} / \mathrm{fm}(K=1)$ has to be viewed in the context of scenario 1 discussed in the introduction which combines parton energy loss with resonance absorption in the fading plasma. Pure parton energy loss in scenario 1 gives a too weak suppression factor to explain experimental data from central $\mathrm{Au}+\mathrm{Au}$-collisions at RHIC.

In scenario 2 without final state absorption, we need roughly $K=8$ or $\hat{q} \simeq 4.0 \mathrm{GeV}^{2} / \mathrm{fm}$ to reproduce the observed nuclear modification factor. If parton energy loss dynamics is the same at LHC, this estimate would lead to a value as large as $\hat{q}=41.9 \mathrm{GeV}^{2} / \mathrm{fm}$ (for $K=8)$. This increase orginates equally from the higher temperature $T=0.5 \mathrm{GeV}$ and the larger scattering cross section of gluons. The values for $\hat{q}$ for both scenarios are summarized in Table 1 .

In our calculation above, we have introduced $K$ as an artifical enhancement parameter. The physical motivation for this soft enhancement factor may come from our estimate of 


\begin{tabular}{|c||c|c|}
\hline$\hat{q}\left[\mathrm{GeV}^{2} / \mathrm{fm}\right]$ & $T=0.3 \mathrm{GeV}$ & $T=0.5 \mathrm{GeV}$ \\
\hline \hline Scenario 1 (parton energy loss + absorption),$K=1$ & 0.5 & 5.2 \\
Scenario 2 (large parton energy loss), $K=8$ & 4.0 & 41.9 \\
\hline
\end{tabular}

Tab. 1: Relevant values of the jet transport parameter $\hat{q}$ in the two scenarios for $T=0.3$ GeV (incident quark) and $T=0.5 \mathrm{GeV}$ (incident gluon). The effective $K$-factor allows possible enhancements of the energy loss parameter $\hat{q}$.

the scattering cross section. The perturbative running coupling may underestimate the soft scattering cross section of the parton in the plasma [32] if one assumes that the temperature defines the relevant scale. More precisely, evaluating the running coupling at $\pi T$ instead of $Q_{0}$ may lead to an enhancement factor of $K \leq 3$ for RHIC temperature. A factor of $K=8$ as in scenario 2 cannot be explained. In contrast, for the higher temperature at LHC the scales $\pi T$ and $Q_{0}$ are approximately equal which makes an enhancement factor $K$ unlikely.

Up to now, the theoretical formalism has focussed on processes at the parton level. In order to compare with observable hadrons, one has to account for hadronization.

\section{Modified Fragmentation Functions $D_{u}^{\pi}, D_{g}^{\pi}$ in the Quark-Gluon Plasma}

For a solution of the modified DGLAP equation (5), we evolve a given fragmentation function $D\left(x, Q_{0}^{2}\right)$ to the ultraviolet scale $Q_{\max }$, i.e. we numerically follow a way opposite to the shower evolution itself. Furthermore, we assume that the fragmentation function at the infrared cut-off scale $Q_{0}$ is unchanged and use standard vacuum fragmentation functions as initial condition. We use the AKK parametrization [14] for the fragmentation of $u$-quarks and gluons into pions

$$
D_{u}^{v, \pi}\left(x, Q_{0}^{2}\right)=0.447 x^{-1.58}(1-x)^{1.01}, \quad D_{g}^{v, \pi}\left(x, Q_{0}^{2}\right)=429 x^{2.00}(1-x)^{5.82}
$$

at the scale $Q_{0}^{2}=2 \mathrm{GeV}^{2}$ in the evolution equations. The numerical solution of the modified DGLAP evolution equation (5) (with and without scattering term) is calculated with the Runge-Kutta method of 4 th order.

At RHIC the fragmentation of quarks is the main source for pion production. To investigate the modification of the $u$-quark to pion fragmentation function in the medium 


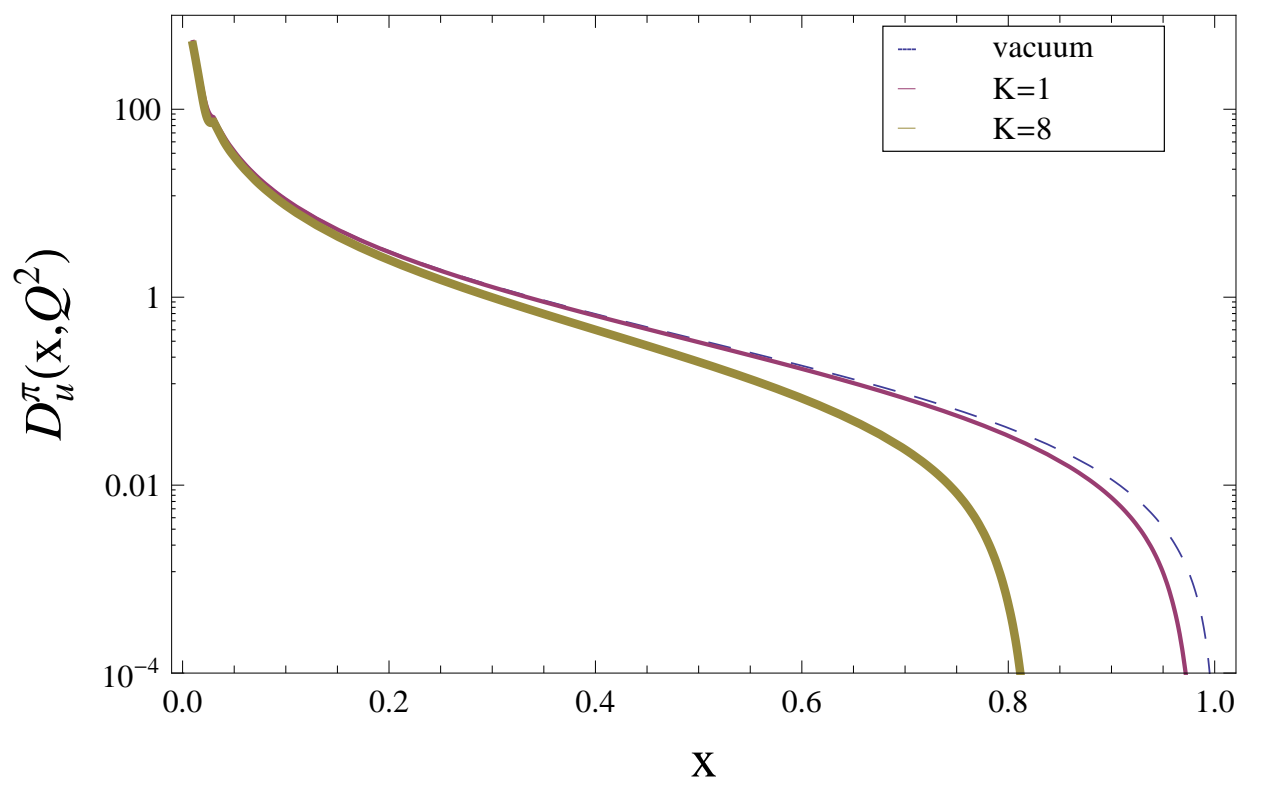

Fig. 1: Fragmentation functions $D_{u}^{\pi}\left(x, Q^{2}\right)$ in medium and vacuum as a function of energy fraction $x$. The plot compares the results for vacuum evolution (dashed curve) to mediummodified evolution with $K=1$ (full drawn thin curve) and $K=8$ (full drawn thick curve). For the calculation, we have used $Q_{\max }=20 \mathrm{GeV}$ and $T=0.3 \mathrm{GeV}$ in Eq. (5)).

at RHIC, we have solved Eq. (5) for $Q_{\max }=20 \mathrm{GeV}$ and $T=0.3 \mathrm{GeV}$. Two different values for the enhancement parameter $K$ are used: $K=1$ and $K=8$.

The results for the gluon to pion fragmentation function are shown in Fig. 1. The overall behaviour of the medium-modified fragmentation function with $Q^{2}$ is similar to the vacuum results. The scattering term acts in a similar manner as the splitting term and "transports" partons from large to small energy fractions. The suppression of leading particles is strongest at large $x$. Following naive expectations, the suppression of the medium fragmentation function becomes stronger for larger $K$.

For LHC, gluon fragmentation is more important than quark fragmentation. The resulting gluon-to-pion fragmentation functions for $Q_{\max }=100 \mathrm{GeV}$ and $T=0.5 \mathrm{GeV}$ are shown in Fig. 2 for both the vacuum case and for the case of a plasma with $K=1$ and $K=8$. Note that the higher temperature at LHC and the larger color charge of the gluon lead to much larger values of $\hat{q}$ (see Table1) although the microscopic dynamics (i.e. energy loss of a fast parton) is unchanged. The scattering term drastically reduces the number of partons with large $x$. This effect is much more pronounced at LHC.

Based on the calculation of modified fragmentation functions, we test the consequences of the two discussed scenarios and compute the nuclear modification factor $R_{A A}$. This 


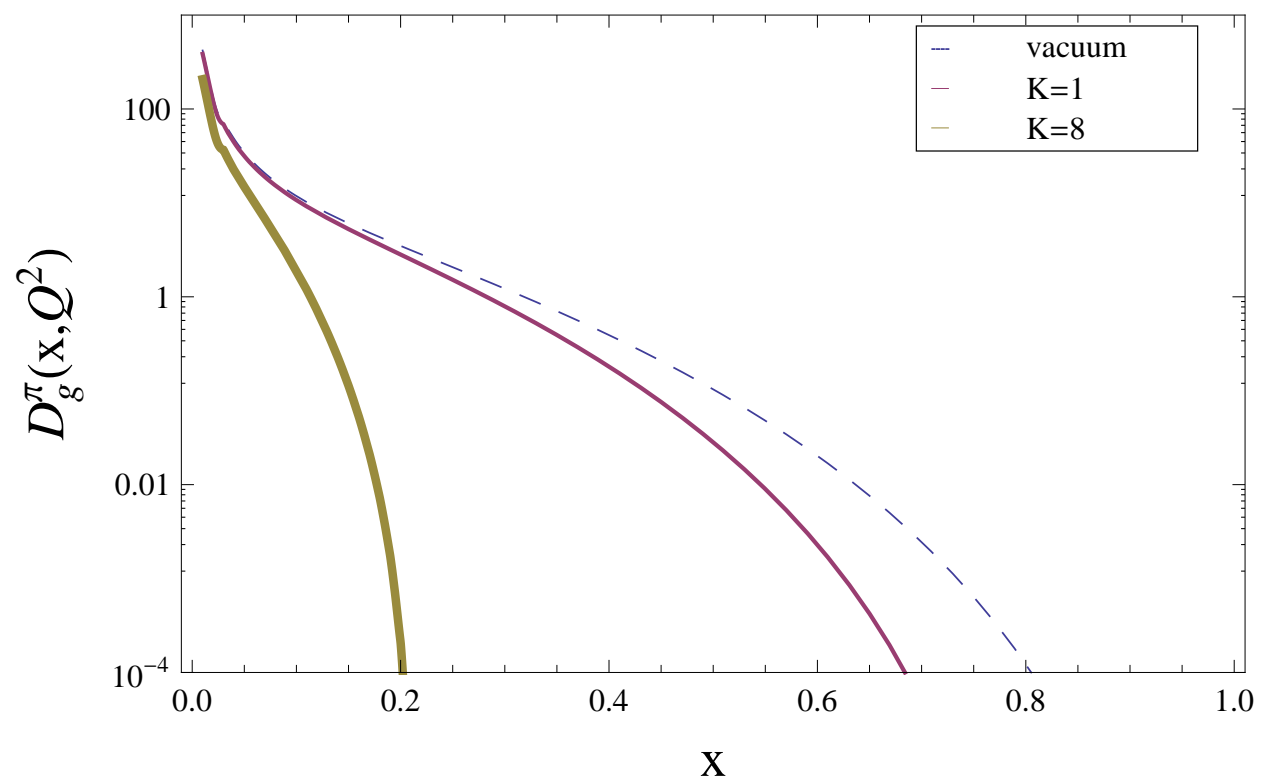

Fig. 2: Fragmentation functions $D_{g}^{\pi}\left(x, Q^{2}\right)$ in medium and vacuum as a function of energy fraction $x$. The plot compares the results for vacuum evolution (dashed curve) to mediummodified evolution with $K=1$ (full drawn thin curve) and $K=8$ (full drawn thick curve). For the calculation, we have used $Q_{\max }=100 \mathrm{GeV}$ and $T=0.5 \mathrm{GeV}$ in Eq. (5)).

experimental observable can be estimated by folding fragmentation functions with the differential cross section $\frac{\mathrm{d} \sigma}{\mathrm{d} q_{\perp}^{2}}$ for the production of the fast parton. For our comparison, we use Pythia 6.4 [15] for a LO calculation of this cross section in $p p$-collisions at $\sqrt{s}=200$ $\mathrm{GeV}$. We can fit the Pythia output with the form

$$
\frac{\mathrm{d} \sigma}{\mathrm{d} q_{\perp}^{2}}=A\left(1+\frac{q_{\perp}^{2}}{1 \mathrm{GeV}^{2}}\right)^{B}\left(1-\frac{2 q_{\perp}}{\sqrt{s}}\right)^{C}
$$

For quark production at RHIC, the parameters have the following values:

$$
A \simeq 468.706 \frac{\mathrm{mb}}{\mathrm{GeV}^{2}}, \quad B \simeq-3.04, \quad C \simeq 9.69
$$

For LHC, the cross section for gluon jet production becomes much larger than for quark jets for typical values of $p_{\perp}$ and consequently our approximation of a gluonic evolution is realistic. In the parametrization for $\frac{\mathrm{d} \sigma}{\mathrm{d} q_{\perp}^{2}}$, we change the parameter values for LHC (with $\sqrt{s}=5.5 \mathrm{TeV}$ ) as follows:

$$
A \simeq 524395 \frac{\mathrm{mb}}{\mathrm{GeV}^{2}}, \quad B \simeq-3.27, \quad C \simeq 10.75
$$


Neglecting initial state effects, the nuclear modification factor can be written as a ratio of the medium fragmentation function folded with the parton cross section to the vacuum fragmentation function folded with the same cross section.

$$
R_{A A}\left(p_{\perp}\right) \simeq \frac{\int \mathrm{d} z \mathrm{~d} q_{\perp}^{2} \frac{\mathrm{d} \sigma}{\mathrm{d} q_{\perp}^{2}} D^{m}\left(z, Q^{2}\right) \delta\left(z q_{\perp}-p_{\perp}\right)}{\int \mathrm{d} z \mathrm{~d} q_{\perp}^{2} \frac{\mathrm{d} \sigma}{\mathrm{d} q_{\perp}^{2}} D^{v}\left(z, Q^{2}\right) \delta\left(z q_{\perp}-p_{\perp}\right)}
$$

The results for $R_{A A}$ in our RHIC scenario for $K=8$ (full drawn thick curve) and $K=1$ ( dashed curve) are shown in Fig. 3. In this plot, it is clearly visible that the natural choice $K=1$ cannot account for RHIC data for $R_{A A}$ (dashed curve), i.e. in scenario 1 parton energy loss alone cannot explain the data. But an artificial tuning of $K$ - and consequently of $\hat{q}$ - to $K=8$ matches the observed nuclear modification factor.

From our perspective, scenario 1 can compete with scenario 2, when an additional mechanism for suppression, namely the absorption of resonances in the resonance gas is included. This additional physics is quite natural, since the lifetime of the plasma and the time for the shower evolution are comparable in magnitude and smaller than the maximum path length allowed by the size of the hot medium. Therefore when the plasma fades out, confinement is no longer an issue and the created resonance gas will allow prehadrons as interaction partners with hadronic cross sections. In the next section, we will discuss in detail this scenario of resonance absorption.

For LHC, we also use Eq. (17) to compute the nuclear modification factor for pions. The result is shown in Fig. 4 for $K=1$ and $K=8$. The larger temperature at LHC leads to an increase of the suppression relative to RHIC. For the case of $K=8$, the computed $R_{A A}$ is $10^{-3}-10^{-5}$ which represents a drastic medium effect. Observing such a large effect at LHC would give strong support for a picture in which the suppression is caused by parton energy loss with a large transport parameter $\hat{q}$.

The other scenario with $K=1$, however, would tell a different story: As discussed in the next section, we expect the absorption of resonances to be much less important at LHC than compared to RHIC. Consequently, the picture of partonic energy loss becomes more realistic and the calculation with $K=1$ becomes closer to reality. In this way, the nuclear modification factor could increase from RHIC to LHC (i.e. less suppression) although the energy loss increases.

\section{Effect of Prehadron absorption in Hot Resonance Matter}

Various time and length scales are involved in the evolution from the quark-gluon plasma to the hadronic phase. Here, we collect numerical values for these time scales to argue that 


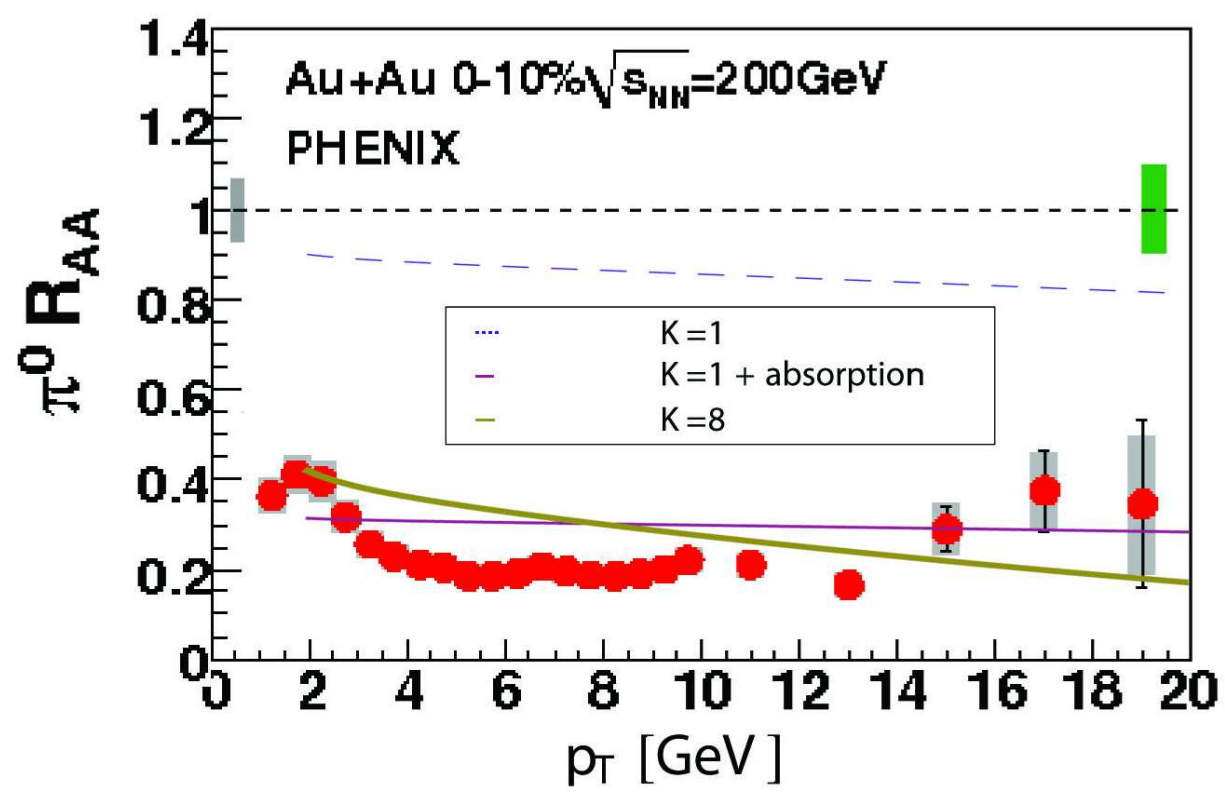

Fig. 3: Two scenarios for the nuclear modification factor $R_{A A}$ of pions at RHIC as a function of their transverse momentum $p_{\perp}$. The full drawn thin curve corresponds to $K=1$ in Eq. (12) while the full drawn thick curve is calculated for a scenario with $K=8$. The dashed curve gives $R_{A A}$ without absorption. For comparison, experimental data from PHENIX [20] are also shown.

absorption plays an important role at RHIC, but is much less relevant at LHC.

We consider the following time scales:

- Equilibration time $\tau_{0}$ of the plasma, which is estimated to be of the order of $0.5 \mathrm{fm}$ at RHIC but much smaller $(<0.2 \mathrm{fm})$ at LHC [2].

- Life time of the plasma which we estimate from longitudinal Bjorken expansion

$$
\tau_{c}=\tau_{0}\left(\frac{T_{0}}{T_{c}}\right)^{3}
$$

For an initial temperature $T_{0}=0.3 \mathrm{GeV}$ at RHIC and $T_{0}=0.5 \mathrm{GeV}$ at LHC, we find $\tau_{c}=3.3 \mathrm{fm}$ at RHIC and $\tau_{c}=6.1 \mathrm{fm}$ at LHC.

- Average time for evolution of the parton from the hard scale $Q_{\max }$ to $Q_{0}$ (where prehadrons can be formed):

$$
\tau_{\text {evo }}=\frac{E}{Q_{0}^{2}}-\frac{E}{Q_{\max }^{2}}
$$

With $E=Q_{\max }=20 \mathrm{GeV}$ for RHIC and $E=Q_{\max }=100 \mathrm{GeV}$ for LHC (and $Q_{0}=\sqrt{2} \mathrm{GeV}$ ), we find $\tau_{\text {evo }}=2 \mathrm{fm}$ for RHIC and $\tau_{\text {evo }}=10 \mathrm{fm}$ for LHC. Note that these time estimate apply to hadrons with average $x$-fraction. 


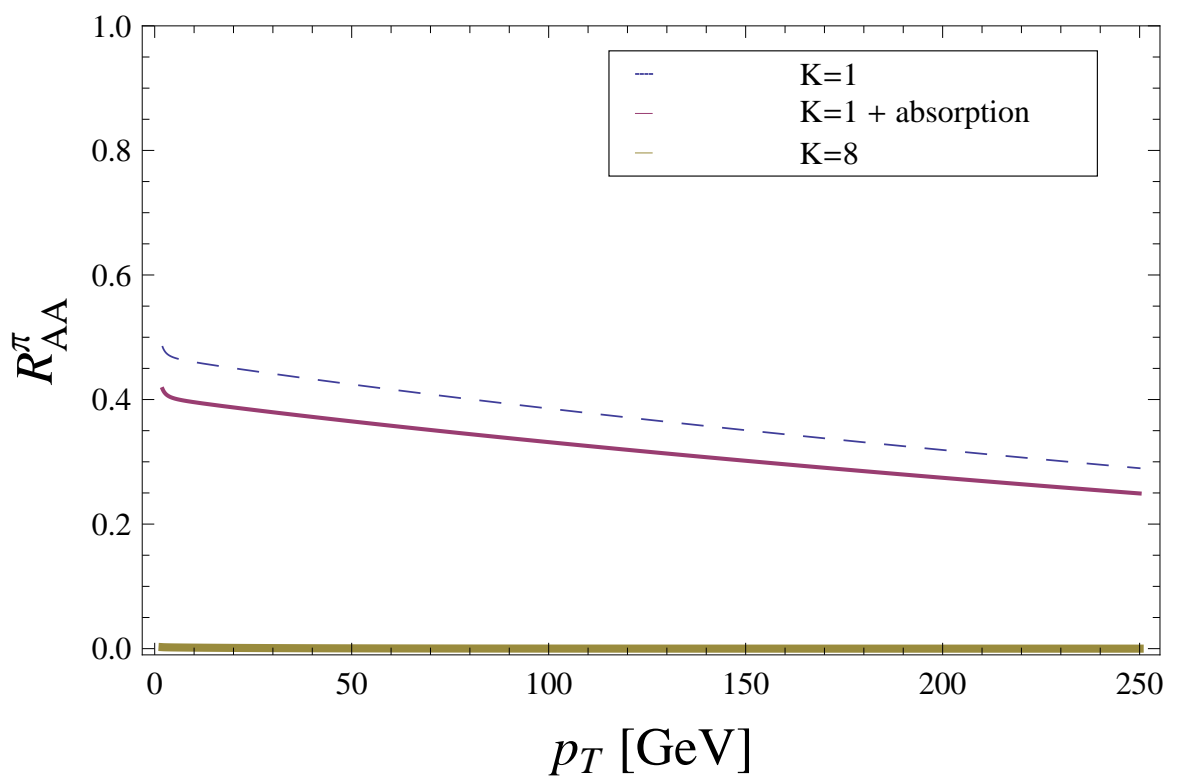

Fig. 4: Two scenarios for the nuclear modification factor $R_{A A}$ of pions at LHC as a function of their transverse momentum $p_{\perp}$. The dashed curve corresponds to $K=1$ in Eq. (12) while the full drawn thin curve is calculated for a scenario with $K=8$. The dashed curve gives $R_{A A}$ without absorption.

- Time in the hot zone $\tau=R / c$ : For central $\mathrm{Au}+\mathrm{Au}$ collisions and central $\mathrm{Pb}+\mathrm{Pb}$ collisions, the size of the plasma is almost identical when estimated in terms of the size of the nuclei involved: $R_{\mathrm{Au}}=6.9 \mathrm{fm}$ and $R_{\mathrm{Pb}}=7.1 \mathrm{fm}$.

The hierarchy between time scales is different at RHIC and LHC. For RHIC, we have the following situation: After the evolution of the shower to the virtuality $Q_{0}$, the plasma has become a resonance gas and preconfined states are formed which can be absorbed in the resonance gas. For an estimate of this effect, we use an exponential form for the nuclear attenuation, averaged over production points $\mathbf{x}_{0}$ and path-length (determined by its angle $\phi_{l}$ of inclination) in a 2-dimensional pancake-like system,

$$
r_{\mathrm{abs}}=\frac{1}{2 \pi} \frac{1}{\pi R^{2}} \int \mathrm{d}^{2} \mathbf{x}_{0} \mathrm{~d} \phi_{l} \frac{1}{2}\left(e^{-n_{\text {res }} \sigma_{\text {res }} l_{1}\left(R, x_{0}, \phi_{l}\right)}+e^{-n_{\text {res }} \sigma_{\text {res }} l_{2}\left(R, x_{0}, \phi_{l}\right)}\right) .
$$

In this equation, $l_{1}$ and $l_{2}$ are the paths of the two partons which are flying in opposite directions in a medium of size $R_{\mathrm{Au}}$ or $R_{\mathrm{Pb}}$ respectively. The two respective path lengths are determined by $\left|\mathbf{x}_{0} \pm\left(l_{i}+\tau_{c}\right) \mathbf{e}_{l}\right|=R$. For the absorption cross section, we use estimates about the resonance gas. These excited hadrons have to deconfine and consequently, their size has to be larger than in vacuum. Therefore, the hadronic cross section is estimated to 
be larger than the $\pi \pi$ cross section (and similar to the $\pi N$ cross section) because of the increased size of the resonances.

$$
\sigma_{\mathrm{res}} \simeq 30 \mathrm{mb}
$$

The above cross section concerns hot matter. In cold matter, the medium consists of unexcited nucleons. Therefore, the formation of the prehadron plays a more important role in the determination of the prehadron-nucleon cross section. Indeed, a cross section reduced by a factor of $2 / 3$ compared to its vacuum value fits the suppression rates of hadrons in deep-inelastic scattering on nuclei [40].

For the density of resonances, we take $n_{\text {res }} \approx T_{c}^{3}$ [12]. The duration of this resonance phase is considerably longer than the plasma phase, since the entropy density has to be dumped into a large volume.

With the estimate given in Eq. (21), one finds values of $r_{\text {abs }} \simeq 0.35$. The final result for the nuclear modification factor, $R_{A A}^{\text {tot }}=R_{A A} r_{\text {abs }}$, is shown in Fig. 3 (full drawn thin curve). This scenario of combined suppression mechanisms can principally account for the large suppression observed in the nuclear modification factor $R_{A A}$ at RHIC.

The situation at LHC is, however, qualitatively different. For a $100 \mathrm{GeV}$ jet, the development of the parton shower takes about $10 \mathrm{fm}$ and we have $\tau_{c}<\tau_{\text {evo. }}$. Consequently, the parton shower will typically not be surrounded by a deconfined medium already at virtuality $Q_{1}>Q_{0}$. Since $\tau_{c} \simeq R$, we can estimate an average value for $Q_{1}$ by demanding

$$
R=\frac{E}{Q_{1}^{2}}-\frac{E}{Q_{\max }^{2}} .
$$

The finite size of the medium can be included by defining two cut-off scales: At $Q_{1}>Q_{0}$ scatterings stop and at $Q_{0}$ perturbative splittings stop. For $Q_{0}<Q<Q_{1}$, we use vacuum evolution equations.

A finite medium length reduces the suppression factor slightly. For a medium of length $R=7 \mathrm{fm}$, the nuclear modification factor roughly increases from 0.4 to 0.6 roughly in the scenario with $K=1$. For the second scenario with $K=8$ (large $\hat{q}$ ), we find $R_{A A} \simeq$ $10^{-2}-10^{-3}$ instead of $10^{-3}-10^{-5}$.

After the evolution to $Q_{0}$, we may also have resonance absorption at LHC. But since $\tau_{\text {evo }} \simeq 10 \mathrm{fm}$ is not much smaller than $2 R$, absorption cannot play a major role. If we let $\tau_{c}$ take the role of $\tau_{\text {evo }}$ in Eq. (20), we find a value of $r_{\text {abs }} \simeq 0.86$ from this equation.

The final result for scenario 1 ( $K=1$ including absorption) for LHC is shown in Fig. 4 (full drawn thin curve). Clearly, absorption is not very important at LHC. 


\section{Modified Parton Distribution in a Jet at small $x$}

In this section, we study the quark/gluon composition of the jet for RHIC/LHC. At small $x$, the presence of the dense and hot medium modifies the peak position of the HumpBacked Plateau in the $\ln (1 / x)$-distribution. As in the previous section, we consider a quark evolution equation in a scenario for RHIC and a gluonic evolution equation for LHC. Here, we use the same scattering term as in the previous section. For the evolution at small $x$, a key ingredient is soft gluon coherence. Without soft gluon coherence, the parton distribution would diverge at small $x$. In order to suppress gluon radiation at small $x$, we solve the evolution equation with the scale $z^{2} Q^{2}$ in the parton distribution [25]

$$
\frac{\partial D\left(x, Q^{2}\right)}{\partial \ln Q^{2}}=\frac{\alpha_{s}\left(Q^{2}\right)}{2 \pi} \int_{x}^{1} \frac{\mathrm{d} z}{z} P(z) D\left(\frac{x}{z}, z^{2} Q^{2}\right)+S\left(x, Q^{2}\right) .
$$

For small $x$, we can reduce the splitting function to $\tilde{P}(z)=2 C_{A} / z$ for gluons (LHC) and $\tilde{P}(z)=2 C_{F} / z$ for quarks (RHIC) since these terms represent the dominant contribution. In the following, we use the splitting function $P(z)=2 C_{R} / z$ to account for both cases. Such an approach is related to the double-logarithmic approximation (DLA) [29] of parton eolution. More terms are taken into account in the framework of MLLA and NMLLA [30, 31.

The scattering term $S\left(x, Q^{2}\right)$ has the same form as in section 2. Its construction and relation to the jet transport parameter $\hat{q}$ is discussed in Eq. (8). Since the thermal gluon mass $m_{s} \propto T$, the scattering term is proportional to $T^{2} / Q^{2}$ which makes it higher twist, a feature shared with the formalisms developed in [33, 22]. Here, we rewrite the scattering term as

$$
S\left(x, Q^{2}\right)=\epsilon \alpha_{s}^{2}\left(Q^{2}\right) \frac{T^{2}}{Q^{2}}\left(D\left(x, Q^{2}\right)+x \frac{\partial D\left(x, Q^{2}\right)}{\partial x}\right)
$$

where

$$
\epsilon=\left\{\begin{array}{cc}
5.4 K & \text { quark jet } \\
12.2 K & \text { gluon jet }
\end{array}\right.
$$

is a dimensionless constant used as an abbreviation for the prefactors. The numerical values are calculated with the help of Eq. (11).

The parameter $K$ allows us to study the two scenarios discussed in the previous section: Scenario $1(K=1)$ includes small parton energy loss and resonance absorption. Scenario $2(K=8)$ parametrizes a large transport parameter $\hat{q}$ tuned up to fit the RHIC data with parton energy loss alone. For the calculation of the parton distribution at small $x$ it is crucial to keep track of the dependence on $\alpha_{s}$. We study the most essential modifications 
and solve the evolution equation in Gaussian approximation [25]. This method reproduces experimental data quite well. The dependence of the scattering term on $\alpha_{s}^{2}$ and the $x$ dependence of the scattering kernel preserves the jet multiplicity, but modifies the position of the maximum and the width of the parton distribution in $\ln (1 / x)$. For further analysis, we define the Mellin transform of $D\left(x, Q^{2}\right)$ by

$$
d\left(J, Q^{2}\right)=\int_{0}^{1} \mathrm{~d} x x^{J-1} D\left(x, Q^{2}\right) .
$$

In Mellin space we can simplify the scattering term by partial integration:

$$
\epsilon \alpha_{s}^{2} \frac{T^{2}}{Q^{2}} \int_{0}^{1} \mathrm{~d} x x^{J-1}\left(D\left(x, Q^{2}\right)+x \frac{\partial D}{\partial x}\left(x, Q^{2}\right)\right)=\epsilon \alpha_{s}^{2} \frac{T^{2}}{Q^{2}}\left(d\left(J, Q^{2}\right)-J d\left(J, Q^{2}\right)\right)
$$

For constant $\alpha_{s}$, the evolution equation reads after Mellin transformation

$$
\frac{\partial d\left(J, Q^{2}\right)}{\partial \ln Q^{2}}=\frac{\alpha_{s}}{2 \pi} \int_{0}^{1} \mathrm{~d} u P(u) d\left(J, u^{2} Q^{2}\right)-\epsilon \alpha_{s}^{2} \frac{T^{2}}{Q^{2}}(J-1) d\left(J, Q^{2}\right) .
$$

We use the following ansatz for the parton distribution in Mellin space with the anomalous dimension $\gamma$

$$
d\left(J, Q^{2}\right) \propto\left(\frac{Q^{2}}{Q_{0}^{2}}\right)^{\gamma\left(J, \alpha_{s}\right)}
$$

for which we obtain a consistency equation $\gamma$

$$
\gamma\left(J, \alpha_{s}\right)=\frac{\alpha_{s} C_{R}}{\pi} \frac{1}{J-1+2 \gamma\left(J, \alpha_{s}\right)}-\epsilon \alpha_{s}^{2} \frac{T^{2}}{Q^{2}}(J-1)
$$

At $J=1$, the contribution from the scattering term vanishes. Since the first moment with $J=1$ of the parton distribution is the jet multiplicity, the multiplicity will not change. This result is natural for a $2 \rightarrow 2$-scattering term. Modifications coming from the scattering term are suppressed since it is a higher-twist $\propto T^{2} / Q^{2}$ contribution. Nevertheless it will turn out that the medium significantly modifies the parton distribution $D\left(x, Q^{2}\right)$.

From Eq. (30) we find for the anomalous dimension:

$$
\gamma\left(J, \alpha_{s}\right)=-(J-1)\left(\frac{1}{4}+\frac{\epsilon \alpha_{s}^{2}}{2} \frac{T^{2}}{Q^{2}}\right)+\sqrt{(J-1)^{2}\left(\frac{1}{4}-\frac{\epsilon \alpha_{s}^{2}}{2} \frac{T^{2}}{Q^{2}}\right)^{2}+\frac{\alpha_{s} C_{R}}{2 \pi}}
$$

The small- $x$ behaviour of the parton distribution is related to its low Mellin moments, therefore we can expand the anomalous dimension around $J=1$.

$$
\gamma\left(J, \alpha_{s}\right) \simeq \sqrt{\frac{\alpha_{s} C_{R}}{2 \pi}}-(J-1)\left(\frac{1}{4}+\frac{\epsilon \alpha_{s}^{2}}{2} \frac{T^{2}}{Q^{2}}\right)+\frac{1}{2} \sqrt{\frac{2 \pi}{\alpha_{s} C_{R}}}(J-1)^{2}\left(\frac{1}{4}-\frac{\epsilon \alpha_{s}^{2}}{2} \frac{T^{2}}{Q^{2}}\right)^{2}
$$


This expansion preserves the most essential features of the parton distribution in vacuum. Since the medium does not change the dominant behavior of the splitting term, it also works in the medium. For running $\alpha_{s}$, we use [25]

$$
d\left(J, Q^{2}\right) \propto \exp \left(\int_{Q_{0}^{2}}^{Q^{2}} \frac{\mathrm{d} Q^{\prime 2}}{Q^{\prime 2}} \gamma\left(J, \alpha_{s}\left(Q^{\prime 2}\right)\right)\right)
$$

For the calculation of the integral, we use the perturbative 1-loop coupling

$$
\alpha_{s}\left(Q^{2}\right)=\frac{1}{b \ln \left(\frac{Q^{2}}{\Lambda_{\mathrm{QCD}}^{2}}\right)}, \quad b=\frac{11-\frac{2 n_{f}}{3}}{4 \pi}, \quad \Lambda_{\mathrm{QCD}}=250 \mathrm{MeV}
$$

The number of flavors is set to five since we study jets with energies well below the mass of the top quark. A calculation with $\alpha_{s}$ in one loop approximation is accurate enough for the vacuum evolution. Note that $d\left(J, Q^{2}\right)$ in Eq. (33) has a Gaussian dependence on $(J-1)$ because the Taylor expansion in Eq. (32) stops at second order:

$$
d\left(J, Q^{2}\right)=C \exp \left(a_{0}+a_{1}(J-1)+a_{2}(J-1)^{2}\right) .
$$

The normalization of $d\left(J, Q^{2}\right)$ is not predicted by the ansatz Eq. (33). We fix the normalization $C \simeq 0.024$ of the multiplicity $n\left(Q^{2}\right)=C \exp \left(a_{0}\right)$ with LEP data from $\mathrm{e}^{+} \mathrm{e}^{-}[27,28]$. It is possible to use these "vacuum" data since the scattering term leaves the multiplicity unchanged.

The coefficients $a_{i}$ in Eq. (35) result from the integration in Eq. (33) and depend on both virtuality and temperature. They are given as follows:

$$
\begin{aligned}
a_{0}= & \frac{1}{b} \sqrt{\frac{2 C_{R}}{\pi \alpha_{s}\left(Q^{2}\right)}}-\left[Q^{2} \rightarrow Q_{0}^{2}\right] \\
a_{1}= & \frac{1}{4 b \alpha_{s}\left(Q^{2}\right)}-\frac{\epsilon}{2 b} \frac{T^{2}}{Q^{2}} \alpha_{s}\left(Q^{2}\right) \\
& -\frac{\epsilon}{2 b^{2}} \frac{T^{2}}{\Lambda^{2}} \operatorname{Ei}\left(-\left(b \alpha_{s}\left(Q^{2}\right)\right)^{-1}\right)-\left[Q^{2} \rightarrow Q_{0}^{2}\right] \\
a_{2}= & \frac{1}{24 b} \sqrt{\frac{\pi}{2 C_{R}}} \alpha_{s}\left(Q^{2}\right)^{-3 / 2}+\frac{1}{2 \sqrt{2} b^{2}} \frac{T^{2}}{\Lambda^{2}} \frac{\pi}{\sqrt{C_{R}}} \operatorname{erf}\left(\left(b \alpha_{s}\left(Q^{2}\right)\right)^{-1 / 2}\right) \epsilon \\
& -\frac{4}{15 b^{3}} \sqrt{\frac{2 \pi}{C_{R}}} \frac{T^{4}}{Q^{4}} \alpha_{s}\left(Q^{2}\right)^{1 / 2} \epsilon^{2}+\frac{1}{2 b} \sqrt{\frac{\pi}{2 C_{R}}} \frac{T^{2}}{Q^{2}} \alpha_{s}\left(Q^{2}\right)^{1 / 2} \epsilon \\
& +\sqrt{\frac{\pi}{2 C_{R}}} \frac{T^{4}}{Q^{4}}\left(\frac{2}{15} \frac{\alpha_{s}\left(Q^{2}\right)^{3 / 2}}{b^{2}}-\frac{1}{10} \frac{\alpha_{s}\left(Q^{2}\right)^{5 / 2}}{b}\right) \epsilon^{2} \\
& -\frac{8}{15 b^{7 / 2}} \frac{T^{4}}{\Lambda^{4}} \frac{\pi}{\sqrt{C_{R}}} \operatorname{erf}\left(\sqrt{\frac{2}{b \alpha_{s}\left(Q^{2}\right)}}\right) \epsilon^{2}-\left[Q^{2} \rightarrow Q_{0}^{2}\right]
\end{aligned}
$$




\begin{tabular}{|c||c|c|}
\hline & vacuum & medium \\
\hline \hline$a_{0}$ & $\frac{1}{b} \sqrt{\frac{2 C_{R}}{\pi \alpha_{s}}}$ & 0 \\
\hline$a_{1}$ & $\frac{1}{4 b \alpha_{s}}$ & $-\frac{\epsilon}{2} \frac{T^{2}}{Q^{2}} \alpha_{s}^{2}$ \\
\hline$a_{2}$ & $\sqrt{\frac{\pi}{2 C_{R}}} \frac{1}{24 b} \alpha_{s}^{-3 / 2}$ & $\frac{\epsilon}{4} \sqrt{\frac{\pi}{2 C_{R}}} \frac{T^{2}}{Q^{2}} \alpha_{s}^{3 / 2}$ \\
\hline
\end{tabular}

Tab. 2: Leading terms of the Gaussian coefficients $a_{0}, a_{1}$ and $a_{2}$ of the parton distribution in a jet in Mellin space, i.e. $\left.d\left(J, Q^{2}\right) \simeq \exp \left(a_{0}+a_{1}(J-1)+a_{2}(J-1)^{2}\right)\right)$, which determine multiplicity $\left(a_{0}\right)$, peak position $\left(a_{1}\right)$ and width $\left(a_{2}\right)$ of the $\ln (1 / x)$-distribution. The first column gives the value in vacuum while the second column shows the correction due to the medium with the lowest power in $\alpha_{s}$. We use a shorthand notation here: One should take $\alpha_{s} \rightarrow \alpha_{s}\left(Q^{2}\right)$ in every term and subtract the same term with $Q^{2} \rightarrow Q_{0}^{2}$.

Here, the notation $-\left[Q^{2} \rightarrow Q_{0}^{2}\right]$ means that one has to subtract the previous terms, replacing $Q^{2}$ by $Q_{0}^{2}$. This subtraction originates from the lower limit of integration in Eq. (33). $\operatorname{Ei}(-z)$ is the exponential integral function, defined by

$$
\operatorname{Ei}(-z)=-\int_{z}^{\infty} \mathrm{d} t \frac{\mathrm{e}^{-t}}{t}
$$

For a better illustration of our results, we also give the lowest-order expansion of the medium modification of the coefficient in $\alpha_{s}$ in Table 2.

The medium modification is solely contained in the modification of the coefficients $a_{i}$. The coefficients $a_{i}$ have the same physical meaning as in vacuum: $a_{0}$ describes the $Q^{2}$-behavior of the jet multiplicity $n\left(Q^{2}\right)=C \exp \left(a_{0}\right), a_{1}$ gives the peak position of the distribution in $\ln (1 / x)$ (see below) and $a_{2}$ is related to the Gaussian width. The first term for each coefficient represents the vacuum result while the second terms proportional to $\epsilon T^{2} / Q^{2}$ represent the modified evolution in Table 2.

In vacuum, the coefficients have inverse powers of $\alpha_{s}\left(Q^{2}\right)$. Therefore the vacuum moments are determined by the upper virtuality $Q^{2}$ with $\alpha_{s}\left(Q^{2}\right)<\alpha_{s}\left(Q_{0}^{2}\right)$. The terms from the medium-modified evolution have positive powers of $\alpha_{s}$ and change the vacuum results. The medium corrections are most important at the infrared scale $Q_{0}^{2}$, where $\alpha_{s}\left(Q_{0}^{2}\right)>\alpha_{s}\left(Q^{2}\right)$. Therefore, in the medium the peak position is shifted to larger values of $\ln (1 / x)$ while the width decreases. 
The well-known MLLA contribution to the peak position,

$$
\Delta a_{1}^{\mathrm{MLLA}}=\frac{1}{2 b}\left(\frac{11 N_{c}}{3}+\frac{2 n_{f}}{3 N_{c}^{2}}\right) \frac{1}{\sqrt{32 N_{c} \pi}} \alpha_{s}\left(Q^{2}\right)^{-1 / 2},
$$

cannot be deduced from the Gaussian approximation and consequently is added by hand. This contribution is subleading but highly relevant for comparison to experimental data.

Finally, the parton distribution can be calculated by an inverse Mellin transformation.

$$
\begin{aligned}
D\left(x, Q^{2}\right) & =\frac{C}{2 \pi i x} \int_{1-i \infty}^{1+i \infty} d J \frac{1}{x^{J-1}} \exp \left(a_{0}-a_{1}(J-1)+a_{2}(J-1)^{2}\right) \\
& =\frac{C}{2 \pi x} \int_{-\infty}^{+\infty} d \tilde{J} \exp \left[a_{0}+i \tilde{J}\left(-a_{1}+\ln \left[\frac{1}{x}\right]\right)-a_{2} \tilde{J}^{2}\right]
\end{aligned}
$$

Performing the Gaussian integral yields

$$
x D\left(x, Q^{2}\right)=\frac{n\left(Q^{2}\right)}{2 \sqrt{\pi a_{2}}} \exp \left(-\frac{\left(\ln \left(\frac{1}{x}\right)-a_{1}\right)^{2}}{4 a_{2}}\right) .
$$

In Fig. 5 we show a comparison of the logarithmic parton distributions $x D\left(x, Q^{2}\right)$ in a jet in vacuum and medium. As discussed after Eq. (25), we use the values $K=1$ and $K=8$ and compare to the vacuum results. Two different kinematical cases are studied: $Q_{\max }=20 \mathrm{GeV}$ and $T=0.3 \mathrm{GeV}$, suitable for RHIC (top), and $Q_{\max }=100 \mathrm{GeV}$ and $T=0.5 \mathrm{GeV}$, relevant for $\mathrm{LHC}$, in the bottom. The shaded area in both figures represents estimates for the region where particles cannot be distinguished from the plasma anymore [23, 24].

At RHIC, the scattering term basically shifts the peak position of the quark distribution to larger $\ln (1 / x)$ (i.e. smaller $x$ ) due to the energy loss from gluon scattering with plasma gluons. The modification of the parton distribution in scenario 1 with $K=1$ is rather small. In contrast, the parton distribution in scenario 2 with $K=8$ differs enormously from the vacuum one. Such a strong modification has not been observed in (preliminary) experimental analyses [23]. Note that for jets the existence of the resonance matter is unimportant.

For LHC, particle identification above the soft background from the plasma is possible over a much wider range. Scenario 2 with $K=8$ leads to a strong modification of the gluon distribution: The peak position is shifted to larger values of $\ln (1 / x)$ and the distribution becomes broader. In contrast, scenario 1 with $K=1$ again leads only to small changes compared to vacuum. 

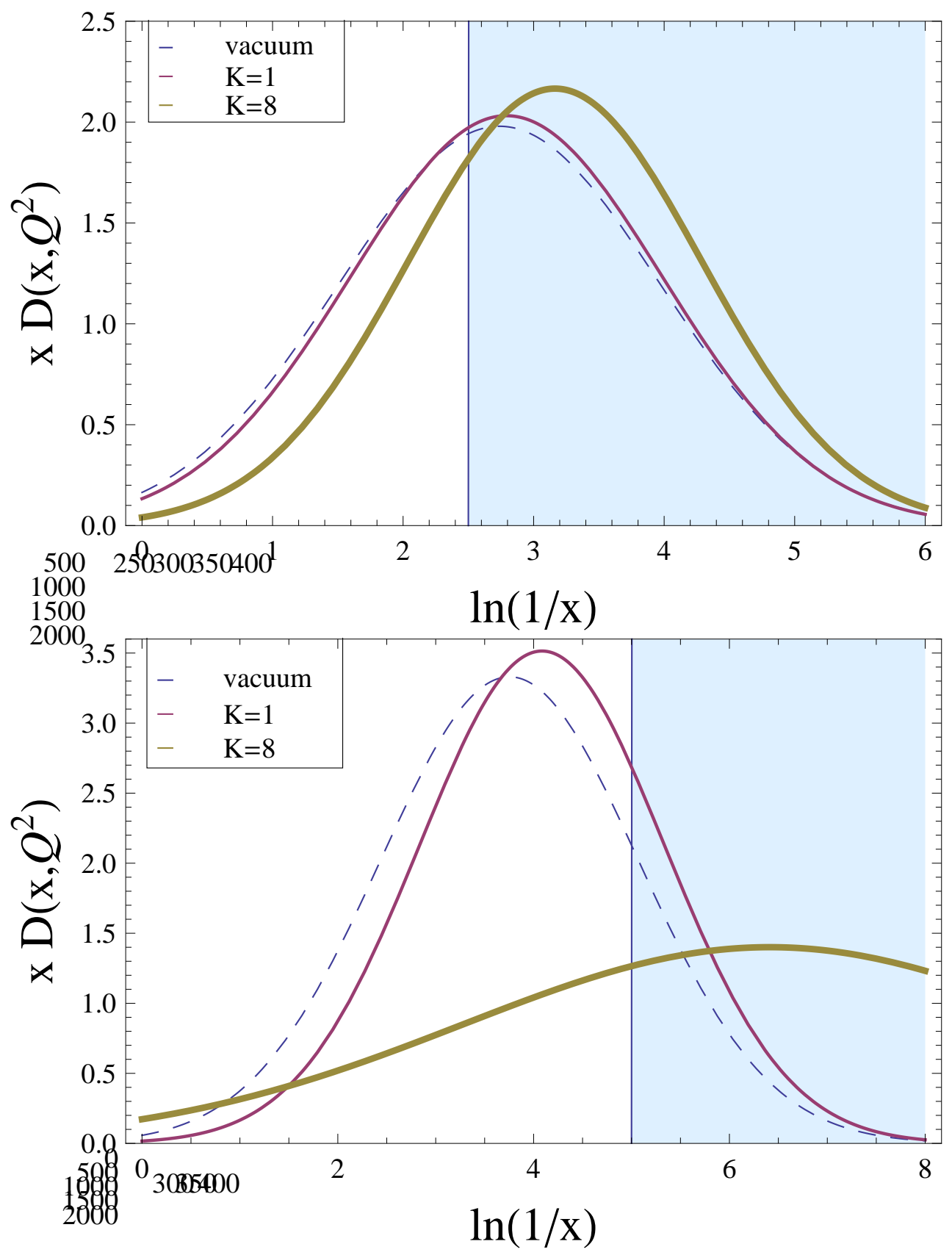

Fig. 5: Top: Quark distribution $x D\left(x, Q^{2}\right)$ in a jet in Gaussian approximation shown for $Q^{2}=$ $(20 \mathrm{GeV})^{2}$ for evolution in vacuum (dashed curve) and medium-modified evolution with $K=1$ (full drawn thin curve) and $K=8$ (full drawn thick curve) in a plasma with temperature $T=$ $0.3 \mathrm{GeV}$ as a function of $\ln (1 / x)$. Bottom: Same for gluons with $Q=100 \mathrm{GeV}$ and $T=0.5$ GeV. 


\section{Summary and discussion}

We have presented two scenarios for jet evolution in nucleus-nucleus collisions. Scenario 1 considers weak parton energy loss caused by scattering in the plasma. After the evolution to a hadronic scale $Q_{0}$, preconfinement causes hadronic absorption in the resonance gas which is left directly after the plasma decay. In contrast, scenario 2 is solely based on strong parton energy loss. In both scenarios, the partonic evolution is computed in the framework of a modified DGLAP equation which combines radiation and scattering of the fast parton in the medium. The corresponding scattering term is of higher twist, $S\left(x, Q^{2}\right) \propto T^{2} / Q^{2}$.

In scenario 1 , we estimate jet transport parameters of $\hat{q} \simeq 0.5 \mathrm{GeV}^{2} / \mathrm{fm}$ at $\mathrm{RHIC}$ and $\hat{q} \simeq 5.2 \mathrm{GeV}^{2} / \mathrm{fm}$ at LHC. Both parameters have to be understood as average values since we do not consider the change of $\hat{q}$ with decreasing temperature and density of the plasma. Enhancement of the QCD estimate $\hat{q} \simeq 0.5 \mathrm{GeV}^{2} / \mathrm{fm}$ by a $K$-factor $K=8$ in scenario 2 can also accomodate our model to the RHIC data for $R_{A A}$. Such an enhanced value may correspond to the hypothesis of a strongly interacting quark-gluon plasma (sQGP).

We do not favour scenario 2, since there are strong evidences for the importance of absorption: (i) Scenario 2 fails to describe production of leading hadrons in deep-inelastic scattering [21], while models including absorption do well [37, 38, 40]; (ii) $J / \Psi$ suppression in the hot medium can be described with a rather small value of the transport coefficient [42], which agrees well with the pQCD expectations; (iii) Even with the enhanced transport coefficient, scenario 2 does not explain the observed strong suppression of open beauty [39], while the effects of absorption make suppression of light and heavy quarks similar [41.

In contrast, we argue that due to the shorter life times of the shower and the plasma at RHIC (compared to the extension of the nuclei), the parton can become preconfined during its way through the medium and interact as a prehadron with the decaying resonance matter. For an estimate of this effect, we use a standard initial density $n_{\text {res }} \approx T_{c}^{3}$ of resonances and an interaction cross section of $\sigma_{\text {res }}=30 \mathrm{mb}$. With these values, we obtain an acceptable fit to the RHIC data with $K=1$.

The new energy domain of LHC allows to test these two alternatives in two different ways. First, we think that due to the similarity of $\alpha_{s}(\pi T)$ and $\alpha_{s}\left(Q_{0}\right)$ for $T=500 \mathrm{MeV}$, $K=1$ is also reasonable for LHC. Then, we predict in scenario 1 a slightly larger $R_{A A}$ due to a stronger partonic energy loss while hadronic absorption plays a minor role. At LHC, the length of the perturbative shower is much longer, leaving almost no time for resonance absorption in the medium after preconfinement. Consequently, prehadron absorption does not increase the suppression factor significantly at LHC. In contrast to scenario 1, scenario 2 would lead to a very large suppression, i.e. $R_{A A}<10^{-2}$ at LHC. Such a strong suppression 
may be detected within the first few months of the heavy-ion program at LHC.

Secondly, one can study the distribution of low- $x$ partons in the jet, since at LHC particles up to $\ln (1 / x) \simeq 5$ are measurable above the plasma background. The shape of the hump-backed plateau of the medium-modified jet will clearly show whether any enhancement of $\hat{q}$ in our calculation is necessary.

For our considerations, we have not made use of further experimental data [43, 44, 45. for several reasons. First, the results from SPS in Ref. [43] and RHIC in Ref. [44] are not fully consistent with each other. The absence of suppression at small energies in [44] is unexpected in our absorption scenario. However, Ref. [43] finds a suppression at a similar energy in the most central collisions. Furthermore, we mention as an aside that the suppression is estimated to set in after a propagation length of $2 \mathrm{fm}$ in [45] which may be related to our estimate for the shower time.

As a final remark, we note that the jet composition in the medium from our calculation can easily be distinguished from approaches with medium-induced gluon radiation [46]. The enhancement of the splitting functions in these calculations always leads to a much higher peak and consequently to a much larger multiplicity [47].

\section{Acknowledgements}

The authors are indebted to K. Reygers for useful discussions on experimental data. S.D. has been supported by the Bundesministerium für Bildung and Forschung (BMBF) under grant number BMBF 06 HD 196 and within the framework of the Excellence Initiative by the German Research Foundation (DFG) through the Heidelberg Graduate School of Fundamental Physics (grant number GSC 129/1). This work was supported in part by Fondecyt (Chile) grant 1090291, by DFG (Germany) grant PI182/3-1, and by ConicytDFG grant No. 084-2009.

\section{References}

[1] S. S. Adler et al. [PHENIX Collaboration], Phys. Rev. Lett. 91 (2003) 072301 arXiv:nucl-ex/0304022.

J. Adams et al. [STAR Collaboration], Phys. Rev. Lett. 91, 172302 (2003) arXiv:nucl-ex/0305015].

[2] I. Arsene et al. [BRAHMS Collaboration], Nucl. Phys. A 757 (2005) 1 arXiv:nucl-ex/0410020]. 
B. B. Back et al., Nucl. Phys. A 757 (2005) 28 arXiv:nucl-ex/0410022].

J. Adams et al. [STAR Collaboration], Nucl. Phys. A 757 (2005) 102 arXiv:nucl-ex/0501009].

K. Adcox et al. [PHENIX Collaboration], Nucl. Phys. A 757 (2005) 184 arXiv:nucl-ex/0410003.

[3] A. Majumder and M. Van Leeuwen, arXiv:1002.2206 [hep-ph].

U. A. Wiedemann, arXiv:0908.2306 [hep-ph].

C. A. Salgado, arXiv:0907.1219 [hep-ph].

D. d'Enterria, arXiv:0902.2011 [nucl-ex].

[4] A. Adare et al. [PHENIX Collaboration], Phys. Rev. Lett. 98 (2007) 172301 arXiv:nucl-ex/0611018.

B. I. Abelev et al. [STAR Collaboration], Phys. Rev. Lett. 98 (2007) 192301 arXiv:nucl-ex/0607012].

[5] R. Baier, Y. L. Dokshitzer, A. H. Mueller, S. Peigne and D. Schiff, Nucl. Phys. B 483 (1997) 291 [arXiv:hep-ph/9607355];

[6] X.N. Wang, Phys. Lett. B 595 (2004), p. 165

Q. Wang and X.N. Wang, Phys. Rev. C 71 (2005), p. 014903

[7] M. Gyulassy, P. Levai and I. Vitev, Nucl. Phys. B 594 (2001), p. 371

M. Gyulassy, P. Levai and I. Vitev, Phys. Rev. Lett. 85 (2000), p. 5535

M. Gyulassy, P. Levai and I. Vitev, Nucl. Phys. B 571 (2000), p. 197

[8] U.A. Wiedemann, Nucl. Phys. B 588 (2000), p. 303

C.A. Salgado and U.A. Wiedemann, Phys. Rev. D 68 (2003), p. 014008

[9] B. G. Zakharov, Phys. Atom. Nucl. 61 (1998) 838 [Yad. Fiz. 61 (1998) 924] arXiv:hep-ph/9807540].

[10] E. V. Shuryak, Nucl. Phys. A 750 (2005) 64 arXiv:hep-ph/0405066].

[11] P. Petreczky, Eur. Phys. J. ST 155 (2008) 123 [arXiv:0711.2280 [hep-lat]].

[12] P. Gerber and H. Leutwyler, Nucl. Phys. B 321 (1989) 387.

[13] L. N. Lipatov, Sov. J. Nucl. Phys. 20 (1975) 94 [Yad. Fiz. 20 (1974) 181].

V. N. Gribov and L. N. Lipatov, Sov. J. Nucl. Phys. 15 (1972) 675 [Yad. Fiz. 15 
(1972) 1218].

G. Altarelli and G. Parisi, Nucl. Phys. B 126, 298 (1977).

Y. L. Dokshitzer, Sov. Phys. JETP 46, 641 (1977) [Zh. Eksp. Teor. Fiz. 73, 1216 (1977)].

[14] S. Albino, B. A. Kniehl and G. Kramer, Nucl. Phys. B 725 (2005) 181 arXiv:hep-ph/0502188.

[15] T. Sjostrand, S. Mrenna and P. Z. Skands, JHEP 0605 (2006) 026 arXiv:hep-ph/0603175.

[16] J. Kapusta, Finite-temperature field theory, Cambridge University Press, 1994

[17] B. Z. Kopeliovich, I. K. Potashnikova and I. Schmidt, arXiv:0707.4302 [nucl-th].

[18] S. A. Bass, C. Gale, A. Majumder, C. Nonaka, G. Y. Qin, T. Renk and J. Ruppert, Phys. Rev. C 79 (2009) 024901 [arXiv:0808.0908 [nucl-th]].

[19] J. Casalderrey-Solana and X. N. Wang, Phys. Rev. C 77 (2008) 024902 arXiv:0705.1352 [hep-ph]].

[20] A. Adare et al. [PHENIX Collaboration], Phys. Rev. Lett. 101, 232301 (2008) arXiv:0801.4020 [nucl-ex]].

[21] W. t. Deng and X. N. Wang, Phys. Rev. C 81, 024902 (2010) arXiv:0910.3403 [hep-ph]].

[22] J. w. Qiu and G. F. Sterman, Nucl. Phys. B 353 (1991) 105.

J. w. Qiu and G. F. Sterman, Nucl. Phys. B 353 (1991) 137.

[23] E. Bruna [STAR Collaboration], arXiv:0905.4763 [nucl-ex].

[24] M. Estienne [ALICE Collaboration], arXiv:0910.2482 [nucl-ex].

[25] R.K. Ellis, W.J. Stirling and B.R. Webber, QCD and Collider Physics, Cambridge University Press 1996.

[26] F. Karsch, K. Redlich and A. Tawfik, Eur. Phys. J. C 29, 549 (2003) arXiv:hep-ph/0303108. 
[27] W. Braunschweig et al. [TASSO Collaboration], Z. Phys. C 47 (1990) 187.

W. Braunschweig et al. [TASSO Collaboration], Z. Phys. C 45 (1989) 193.

D. Decamp et al. [ALEPH Collaboration], Phys. Lett. B 273 (1991) 181.

P. Abreu et al. [DELPHI Collaboration], Z. Phys. C 50 (1991) 185.

M. Z. Akrawy et al. [OPAL Collaboration], Phys. Lett. B 247 (1990) 617.

B. Adeva et al. [L3 Collaboration], Phys. Lett. B 259 (1991) 199.

[28] M. Schmelling, Phys. Scripta 51 (1995) 683.

[29] Yu. Dokshitzer, V.A. Khoze, Basics of perturbative QCD, Edition Frontieres 1991

[30] R. Perez-Ramos, F. Arleo and B. Machet, Phys. Rev. D 78 (2008) 014019 arXiv:0712.2212 [hep-ph]].

[31] R. P. Ramos, JHEP 0609 (2006) 014 arXiv:hep-ph/0607223.

R. P. Ramos, JHEP 0606 (2006) 019 arXiv:hep-ph/0605083.

[32] J. Braun and H. J. Pirner, Phys. Rev. D 75, 054031 (2007) arXiv:hep-ph/0610331.

[33] X. N. Wang and X. f. Guo, Nucl. Phys. A 696 (2001) 788 arXiv:hep-ph/0102230].

[34] Y. Maezawa et al. [WHOT-QCD Collaboration], Nucl. Phys. A 830 (2009) 247C arXiv:0907.4203 [hep-lat]].

[35] S. Domdey, G. Ingelman, H. J. Pirner, J. Rathsman, J. Stachel and K. Zapp, Nucl. Phys. A 808 (2008) 178 [arXiv:0802.3282 [hep-ph]].

S. Domdey, PhD thesis

[36] F. Arleo, Eur. Phys. J. C 61 (2009) 603 [arXiv:0810.1193 [hep-ph]].

[37] B.Z. Kopeliovich, J. Nemchik and E. Predazzi, Proceedings of the workshop on Future Physics at HERA, ed. by G. Ingelman, A. De Roeck and R. Klanner, DESY 1995/1996, v. 2, 1038; arXiv: nucl-th/9607036.

[38] B. Z. Kopeliovich, J. Nemchik, E. Predazzi and A. Hayashigaki, Nucl. Phys. A 740, 211 (2004).

[39] M. Djordjevic, M. Gyulassy and S. Wicks, Phys. Rev. Lett. 94, 112301 (2005) arXiv:hep-ph/0410372. 
[40] A. Accardi, D. Grunewald, V. Muccifora and H. J. Pirner, Nucl. Phys. A 761 (2005) 67 arXiv:hep-ph/0502072.

A. Accardi, V. Muccifora and H. J. Pirner, Nucl. Phys. A 720 (2003) 131 arXiv:nucl-th/0211011].

[41] B. Z. Kopeliovich and J. Nemchik, arXiv:1009.1162 [hep-ph].

[42] B. Z. Kopeliovich, I. K. Potashnikova and I. Schmidt, Phys. Rev. C 82 (2010) 024901 arXiv:1006.3042 [nucl-th]].

[43] M. M. Aggarwal et al. [WA98 Collaboration], Phys. Rev. Lett. 100, 242301 (2008) arXiv:0708.2630 [nucl-ex]].

[44] A. Adare et al. [PHENIX Collaboration], Phys. Rev. Lett. 101, 162301 (2008) arXiv:0801.4555 [nucl-ex]].

[45] S. S. Adler et al. [PHENIX Collaboration], Phys. Rev. C 76, 034904 (2007) arXiv:nucl-ex/0611007.

[46] N. Borghini and U. A. Wiedemann, arXiv:hep-ph/0506218.

[47] R. P. Ramos, Eur. Phys. J. C 62 (2009) 541 [arXiv:0811.2418 [hep-ph]]. 